\title{
Orbitofrontal cortex neurofeedback produces lasting changes in contamination anxiety and resting-state connectivity
}

\author{
D Scheinost ${ }^{1}$, T Stoica ${ }^{2}$, J Saksa ${ }^{3}$, X Papademetris ${ }^{1,2}$, RT Constable ${ }^{1,2,4}$, C Pittenger ${ }^{3,5,6}$ and M Hampson ${ }^{2}$
}

Anxiety is a core human emotion but can become pathologically dysregulated. We used functional magnetic resonance imaging (fMRI) neurofeedback (NF) to noninvasively alter patterns of brain connectivity, as measured by resting-state fMRI, and to reduce contamination anxiety. Activity of a region of the orbitofrontal cortex associated with contamination anxiety was measured in real time and provided to subjects with significant but subclinical anxiety as a NF signal, permitting them to learn to modulate the target brain region. NF altered network connectivity of brain regions involved in anxiety regulation: subjects exhibited reduced restingstate connectivity in limbic circuitry and increased connectivity in the dorsolateral prefrontal cortex. NF has been shown to alter brain connectivity in other contexts, but it has been unclear whether these changes persist; critically, we observed changes in connectivity several days after the completion of NF training, demonstrating that such training can lead to lasting modifications of brain functional architecture. Training also increased subjects' control over contamination anxiety several days after the completion of NF training. Changes in resting-state connectivity in the target orbitofrontal region correlated with these improvements in anxiety. Matched subjects undergoing a sham feedback control task showed neither a reorganization of restingstate functional connectivity nor an improvement in anxiety. These data suggest that NF can enable enhanced control over anxiety by persistently reorganizing relevant brain networks and thus support the potential of NF as a clinically useful therapy.

Translational Psychiatry (2013) 3, e250; doi:10.1038/tp.2013.24; published online 30 April 2013

\section{Introduction}

Normal and pathological patterns of behavior and thought correspond to the activity of particular brain circuits. Interventions that alter patterns of behavior and thought therefore must act on the organization of the underlying circuits; some clinical interventions, such as deep brain stimulation, do so explicitly through anatomically targeted manipulations of brain function. ${ }^{1}$ The ability to manipulate targeted brain circuits of relevance to particular behavior patterns in a non-invasive manner would be of immense interest and clinical utility.

Poorly controlled anxiety reduces the quality of life of many healthy individuals and is a key symptom of numerous neuropsychiatric conditions. Contamination anxiety, in particular, is prevalent in the healthy population and is a common symptom in obsessive-compulsive disorder (OCD). ${ }^{2}$ Pharmacological and behavioral interventions are widely used in the treatment of anxiety and of OCD, but for many individuals these are of little efficacy or are associated with troublesome side effects. In extreme cases, invasive anatomically targeted interventions are sometimes used for $O C D$ and can be effective. ${ }^{1}$

Neurofeedback (NF) describes the process of learning to control neural processes via an explicit feedback signal. Realtime functional magnetic resonance imaging (rt-fMRI) NF is a novel approach in which subjects can receive direct feedback regarding neural activity, as reflected in the BOLD signal, of a defined brain region. Recent studies have reported success in training subjects to manipulate activity in specific target brain regions using this approach. ${ }^{3-5}$ Several studies have also reported that such training can translate into changes in behavioral measures ${ }^{6-9}$ or clinical symptoms. ${ }^{10,11}$ Learned control over a specific region of the brain has been shown to lead to alterations in brain networks, with documented alterations in brain function lasting only during ${ }^{3}$ or immediately after the presentation of feedback. ${ }^{5,12}$ For NF-induced changes in brain networks to be of clinical utility, it is essential that such changes persist beyond the scanning session in which training occurred.

We asked whether NF can produce functional alterations in the circuitry associated with contamination anxiety, thereby reducing it, and whether such changes can persist over the days following training. Activation in the orbitofrontal cortex (OFC) has been implicated in contamination anxiety in healthy individuals ${ }^{13}$ and in patients with OCD. ${ }^{14}$ We hypothesized that feedback training that permitted subjects to manipulate neural activity in the OFC would result in a reorganization of associated functional brain networks, as measured by resting-state $\mathrm{fMRl}$, and in a reduction in experienced anxiety. We investigated this in individuals with significant contamination anxiety but without any clinical diagnosis of an anxiety disorder.

\footnotetext{
${ }^{1}$ Department of Biomedical Engineering, Yale University, New Haven, CT, USA; ${ }^{2}$ Department of Diagnostic Radiology, Yale School of Medicine, New Haven, CT, USA; ${ }^{3}$ Department of Psychiatry, Yale School of Medicine, New Haven, CT, USA; ${ }^{4}$ Department of Neurosurgery, Yale School of Medicine, New Haven, CT, USA; ${ }^{5}$ Department of Psychology, Yale University, New Haven, CT, USA and ${ }^{6}$ Child Study Center, Yale School of Medicine, New Haven, CT, USA

Correspondence: Dr M Hampson, Magnetic Resonance Research Center (MRRC), Yale School of Medicine, The Anlyan Center, N121, 300 Cedar Street, PO Box 208043, New Haven, CT 06520-8043, USA.

E-mail: michelle.hampson@yale.edu

Keywords: contamination anxiety; neurofeedback; obsessive-compulsive disorder; real-time fMRI; resting connectivity

Received 26 November 2012; revised 24 January 2013; accepted 18 February 2013
} 


\section{Materials and methods}

Subject recruitment. Subjects with high contaminationrelated anxiety were recruited and consented in accordance with a protocol reviewed and approved by the Yale University Human Research Protection Program. Individuals with a history of neuropsychiatric disorder or currently using medication (other than antibiotics or birth control) were excluded from the study. All included subjects had a score of $\geqslant 8$ on the Padua Inventory-Washington State University Revision ${ }^{15}$ - Contamination Obsessions and Washing Compulsions subscale. A total of 12 subjects were recruited for the NF group, and 11 subjects, matched for age and gender, were recruited for the sham-feedback (SF) control group. One NF subject was removed from analysis for not following instructions and was not matched with a SF subject. Further, one NF subject and the matched SF subject were removed from analysis due to an error in the localization of the target region discovered after their data collection (but before assessment of imaging outcome measures). A total of $10 \mathrm{NF}$ and 10 matched SF subjects were included in the final analysis (4 females in each group). Each of the 20 subjects had four separate scanning sessions over the course of 3 weeks (80 scanning session in total were analyzed). There was no significant difference in age or scores on the Padua Contamination subscale between these two groups.

Study protocol. Our rt-fMRI NF protocol has been described in detail in a methods publication ${ }^{16}$ using the system described in Scheinost et al. ${ }^{17}$ We provide here a general description but refer the reader there for more specific information. Briefly, the experimental paradigm consisted of four MRI scanning sessions, spaced roughly half a week apart. The first visit began with an imaging session involving high-resolution anatomical images, two resting-state functional runs ( $5 \mathrm{~min}$ each) and a functional localizer that alternately presented neutral and contamination-related images. The localizer was used to identify the target region of interest, defined as the 30 OFC voxels, bilaterally, that were maximally differentially activated by contamination-related stimuli. After this first imaging session, subjects met with a clinical psychologist to help them develop initial strategies for manipulating their brain activity that could later be refined via NF. The second visit began with an out-ofmagnet assessment of how well subjects could control their anxiety. In this assessment session, subjects viewed 25 contamination-related images and were instructed to try to minimize their anxiety in response to each image and to indicate on a 1-5 scale the anxiety they experienced. Subjects then participated in a 90-min NF (or SF) imaging session involving 10 functional runs during which they tried to control brain activity, as reported to them in a line graph on the visual display, while viewing contamination-related and neutral images (as further described below). The first and last two runs were performed without NF; the middle six were performed with NF. During the NF runs, subjects were instructed to try to learn what worked the best for controlling their OFC activity. During the non-NF runs, subjects were instructed to do whatever they felt worked best for controlling OFC activity. The non-NF runs, referred to as control task runs, were used to assess changes in the OFC in the absence of feedback. The third visit was identical to the second. The fourth visit consisted of a final assessment of anxiety evoked by contamination-associated stimuli and a final imaging session in which two resting-state runs were collected to assess changes in functional connectivity. The precise scheduling of the visits varied based on availability of scan slots, but the final visit was always scheduled 1-7 days after the third visit. The average was 3 days, and there was no significant difference between the sham and real NF subjects in this variable. As the final visit, in which we assessed the effects of the NF on both anxiety and brain functional connectivity, occurred several days after feedback training, the observed effects do not reflect transient results of feedback training but rather reflect changes that persisted for several days. In total, each subject participated in four MRI sessions during the experiment and $>80$ scanning sessions were performed in this experiment.

Subjects never viewed the same stimulus twice. The sets of contamination and neutral images used at different time points in the protocol were balanced for induced anxiety, as described previously. ${ }^{16}$

Task during NF sessions. During NF, subjects viewed an arrow on the left side of the screen that cued them regarding the current task. A red up-arrow indicated they should try to increase activity in their OFC, a blue down-arrow indicated they should try to decrease activity in the region, and a white forward-arrow indicated they should rest and not try to control the region. To the right of this arrow, a large contamination-related image was shown during the increase and decrease conditions and a neutral image was shown during the rest condition. The arrows and images changed every $26 \mathrm{~s}$, cycling through the three conditions. During NF runs, a line graph was included at the bottom of the screen indicating the activity in the subject's OFC. The line was color-coded to indicate the current task and was updated after each newly acquired volume was analyzed, approximately every $2 \mathrm{~s}$. Although the NF group viewed the actual time course of the OFC, the SF group viewed the time course of the corresponding run performed previously of their paired NF subject. As a result, the SF subjects viewed exactly the same stimuli as their paired NF subjects. An example of what subjects viewed during the NF runs is provided in Figure 1.

MR imaging protocol. All imaging was done on a 1.5-T Siemens Sonata scanner (Siemens Medical Systems, Erlangen, Germany). A sequence designed to optimize signal in the OFC was used for all functional data collection (repetition time $=2000 \mathrm{~ms}$, echo time $=30 \mathrm{~ms}$, flip angle $=80$, bandwidth $=2604,200 \mathrm{~mm}$ field of view for $3.1 \mathrm{~mm}$ isotropic voxels, 31 axial-oblique slices covering the OFC and brain above).

Preprocessing. Images were slice time corrected using sinc interpolation in Matlab (www.mathworks.com) and motion corrected using SPM5 (http://www.fil.ion.ucl.ac.uk/ spm/software/spm5/). Unless noted, all analyses were conducted using Biolmage Suite. ${ }^{18}$ 


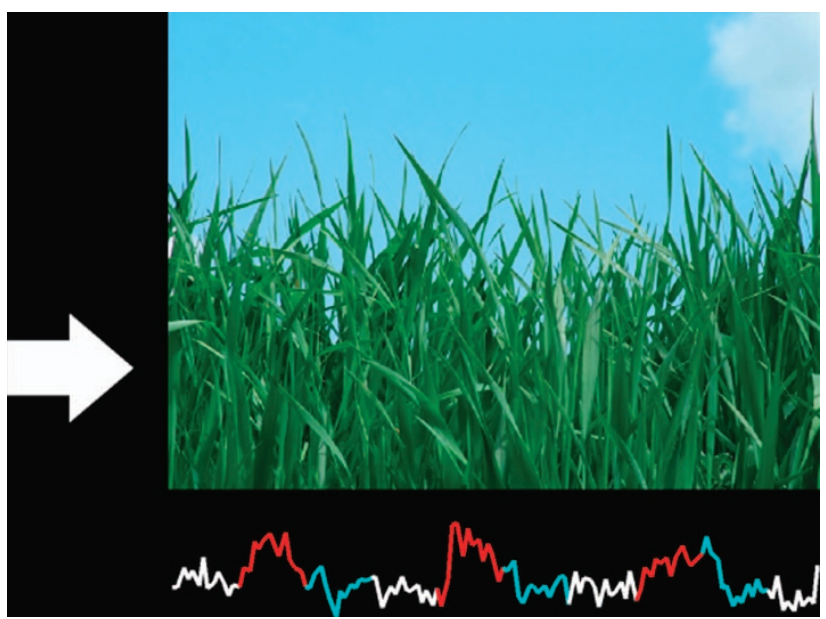

Figure 1 A screen-shot showing the visual display at the end of a neurofeedback run, which ended with a rest block and a corresponding neutral image. The images shown during increase and decrease blocks were designed to induce contamination anxiety. The time course of the orbitofrontal cortex, shown at the bottom, tended to be higher in the red relative to the blue periods, indicating some control over their target region.

Resting-state connectivity analysis. Several covariates of no interest were regressed from the data, including linear and quadratic drift, six rigid-body motion parameters and mean cerebral-spinal fluid, white matter and global signals. The data were low-pass filtered (approximate cutoff frequency $=0.12$ $\mathrm{Hz}$ ) and a gray matter mask was applied to the data so that only voxels in the gray matter were included. The network measure of degree ${ }^{19,20}$ was computed for each voxel. Comparison of degree maps before and after NF allows exploration of functional connectivity changes, unbiased by $a$ priori assumptions regarding regions of interest. First, the time series for a voxel was correlated with every other voxel in the gray matter with degree defined as the number of connection with a correlation greater than $r=0.40$. The process was repeated for each voxel. Degree maps were normalized as described previously.21,22 For group comparisons, single subject results were smoothed (6 $\mathrm{mm}$ Gaussian kernel) and warped to common space through a series of linear and nonlinear registrations as described previously. ${ }^{19,22}$

Control task analysis. Control task data collected immediately before and after NF on the second and third experimental days were used to assess changes in the target region of interest. Task regressors for increase and decrease blocks were convolved with a hemodynamic response function and included in the design matrix of a general linear model. Motion parameters and drift terms were added as regressors of no interest. Activity in the target OFC voxels during decrease blocks was subtracted from activity during increase blocks to yield an estimate of control over the region during control task runs.

\section{Statistical analyses}

Resting connectivity data. Group level statistics were performed in a voxel-wise manner to identify regions showing changes in functional connectivity after rt-fMRI training. For each group, paired differences in the resting data collected before and after training were identified using Wilcoxon's signed-rank test. Between-group differences were identified using Wilcoxon's rank-sum test, implemented in Analysis of Functional Neurolmages (AFNI) (http://afni.nimh.nih.gov/ afni). To assess the relationship between behavior and intrinsic connectivity patterns, as previous studies have done, ${ }^{23}$ we performed a voxel-by-voxel Pearson correlation analysis between the change in behavior and change in degree. Significance was assessed at a $P<0.05$ level after correcting for multiple comparisons across the grey matter via AFNI's AlphaSim program. To check for possible confounds caused by group differences in head motion, ${ }^{24}$ we computed the mean frame-to-frame displacement for each subject across all time points. Wilcoxon's rank-sum tests revealed no group difference in motion $(P=0.67$, $U=44)$ and no difference in motion between the first and last imaging session $(P=0.57, U=42)$.

Behavioral data. The anxiety ratings were averaged across stimuli in each assessment session. The average in the final session was subtracted from the average in the first session to yield an estimate of how much each subject increased control over their contamination anxiety. Wilcoxon's signedrank tests were used to assess changes within each group, and a Wilcoxon's rank-sum test was used to contrast changes between groups. Because we hypothesized a priori that feedback subjects would increase control over their anxiety, and would have a greater increase in control than the sham subjects, significance was assessed using onetailed tests at a $P<0.05$ level.

Control task data. Within-group changes in OFC (percentage of signal change at the final time point compared with percentage of signal change at the first time point) were tested with Wilcoxon's signed-rank tests and between-group differences were tested with a Wilcoxon's rank-sum test. Because we hypothesized a priori that feedback subjects would increase control over their OFC activity, and would have a greater increase in control than the sham subjects, significance was assessed using one-tailed tests at a $P<0.05$ level.

\section{Results}

Changes in resting state functional connectivity patterns before and after NF were assessed using a network theory metric, degree of connectivity, computed on a voxel-wise basis. ${ }^{20}$ The group composite map of whole-brain changes in degree of connectivity in the feedback group showed significant $(P<0.05$ corrected) decreases in brain regions associated with emotion processing, including the insula and adjacent regions, the hippocampi, parahippocampal and entorhinal cortex, the right amygdala, the brainstem in the vicinity of the substantia nigra, the temporal pole, superior temporal sulcus, thalamus and fusiform gyrus. By contrast, significantly $(P<0.05$ corrected) increased degree of connectivity was seen in prefrontal areas associated with emotion regulation and cognitive control, ${ }^{25}$ including right lateral prefrontal cortex and bilateral portions of Brodmann's area 8 (Figure 2a). The composite map from the sham group did not show any loci of significant changes in connectivity surviving multiple comparisons correction (not 

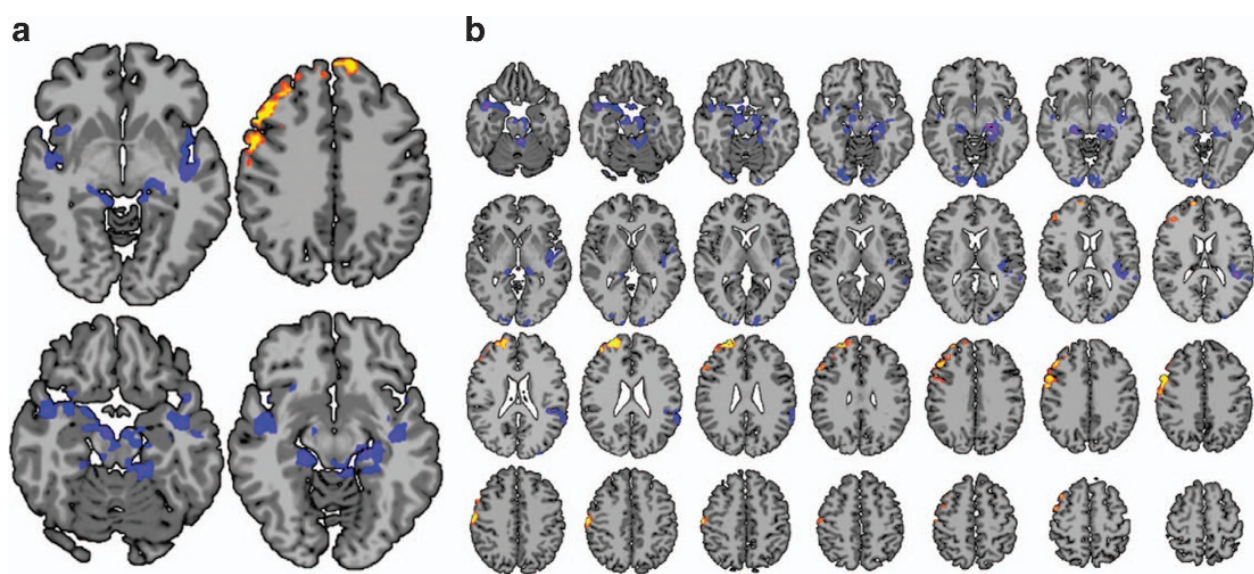

Figure 2 (a) Changes in degree of connectivity in the feedback group. Increases are shown in red/yellow and decreases in blue/purple. Decreases in connectivity are seen in limbic areas, and increases are seen in prefrontal regions. (b) Contrast between the feedback and sham groups. This contrast is similar to the feedback group composite map, suggesting that the changes in connectivity in the feedback group were a result of the feedback rather than habituation or some other non-specific aspect of training. All slices are shown with radiological convention (left is on the right) at a whole-brain-corrected $P<0.05$ threshold.

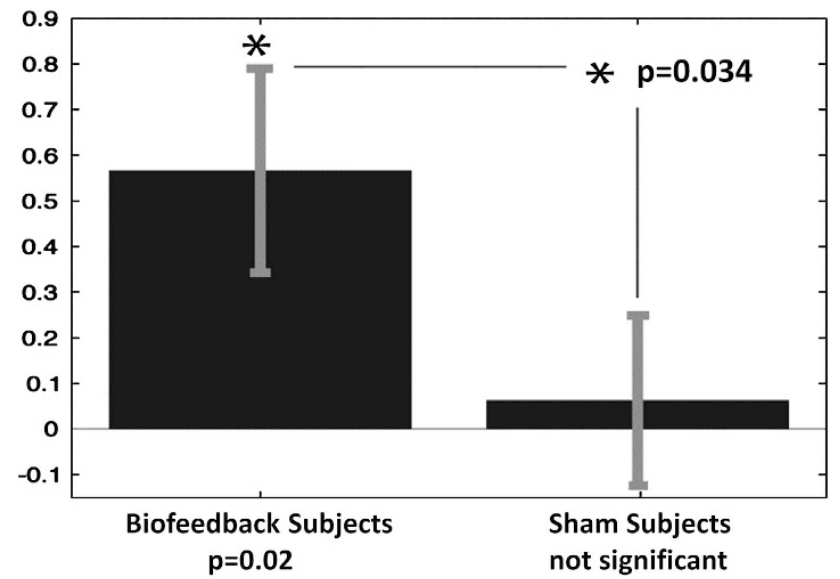

Figure 3 Change in control over contamination anxiety in the two groups. The neurofeedback subjects significantly increased their control over anxiety (indicated by an asterisk) and the sham subjects did not.

shown). The contrast between the two groups at $P<0.05$ corrected was similar to the feedback group composite map (Figure 2b), further suggesting that the changes in connectivity were due specifically to the feedback and not to non-specific aspects of the task.

Control over contamination anxiety was assessed by showing subjects contamination-associated images before and after NF and instructing them to control their anxiety and report how much anxiety they experienced. NF subjects showed a significant reduction in contamination anxiety several days following the feedback sessions $(P=0.02$, median $=0.27$ ), based on these self-report measures, while sham subjects did not $(P=0.45$, median $=0.04)$. There was a significant difference between the groups $(P=0.034$, $U=25.5$; Figure 3 ), confirming that the improvement in the intervention group was due to the feedback rather than to habituation or some other non-specific aspect of training.

To identify the specific connectivity changes most associated with improved control over anxiety, we created a map for the feedback group of the correlation between changes in degree of connectivity (Figure 2a) and increased control over anxiety (Figure 3 ). Two areas of significant correlation $(P<0.05$ corrected) are apparent: the target region of the OFC, bilaterally, and a right lateral parietal area (Figure 4).

Changes in control over OFC activity were assessed in the absence of a NF signal at the start and end of each of the two 90-min feedback sessions. Comparison of the first and final assessments revealed that the NF subjects changed activity in the target area of their OFC $(P<0.01$, median $=0.2689)$, while the sham subjects did not $(P=0.43$, median $=$ -0.0642 ; Figure 5). The contrast between groups approached statistical significance $(P=0.07, U=30)$.

\section{Discussion}

Mitigation of maladaptive behavioral states entails inducing change in the underlying brain circuitry. We demonstrate that rt-fMRI NF can modulate intrinsic brain connectivity patterns associated with anxiety control, thereby enhancing control over contamination anxiety.

These results extend previous applications of rt-fMRI NF in two critical ways. First, we show changes in both anxiety regulation and brain connectivity that persist for several days following the end of NF training. Previous rt-fMRI studies have reported success in training subjects using specific target brain regions $s^{4,26}$ and in altering relevant connectivity patterns during task performance ${ }^{5,27}$ or at rest. ${ }^{12}$ However, these changes in brain network function have been reported only during NF or immediately after it and may therefore represent state changes, rather than lasting alterations in brain functional architecture. Transient symptomatic improvements or changes in brain network connectivity would clearly be of only limited clinical utility and interest. We demonstrate that rt-fMRI training can induce changes in both resting-state brain patterns and behavior that last for days after the last NF session: both the post-feedback resting-state data and the post-intervention behavioral data were collected several days after the completion of the NF intervention. Therefore, the increased control over anxiety produced by NF (Figure 3) reflects a persistent change, not an acute consequence of the training. Similarly, the changes in connectivity we observe 

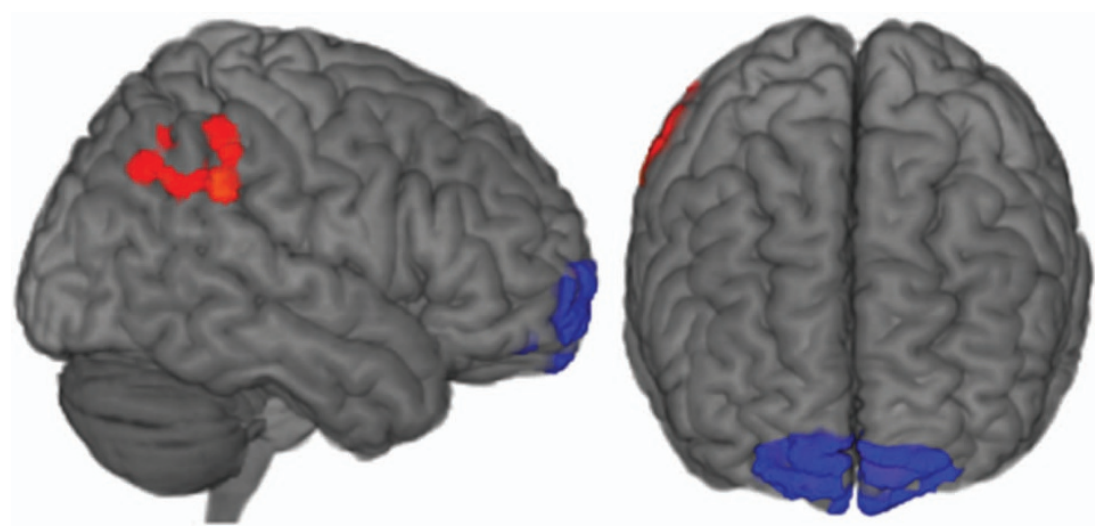

Figure 4 Regions of the brain where changes in degree of connectivity correlated with increased control over contamination anxiety, at a whole-brain-corrected $P<0.05$ threshold. Positive correlations between increased control over anxiety and increased degree of connectivity are shown in red/yellow and negative correlations are shown in blue/purple. Increased control over anxiety was associated with decreased connectivity in the orbitofrontal cortex and increased connectivity in a right parietal region.

(Figures 2a, b and 4) reflect persistent alterations produced by the intervention. These lasting alterations suggest that rt-fMRI is a promising mechanism to induce clinically meaningful changes in the brain.

Second, we show that this approach can be used to modulate a particular form of anxiety. Anxiety is a core symptom of many psychiatric conditions and a substantial source of distress in both clinical and non-clinical populations. Contamination anxiety, in particular, is a cardinal symptom of OCD and is common in the non-clinical population, ${ }^{2}$ such as the subjects described here. The ability of rt-fMRI NF to modulate this core emotional state creates clear possibilities for clinical applications.

The observed alterations in resting-state connectivity in the feedback group (Figures $2 a$ and $b$ ) show that rt-fMRI is capable of reorganizing the functional brain architecture associated with emotion processing. The pattern of change in functional connectivity, with decreased connectivity in limbic and paralimbic areas and increased connectivity in lateral and anterior frontal areas associated with cognitive control, is consistent with a model in which dorsolateral prefrontal cortical areas have a role in emotional control by downregulating the function of areas involved in emotion generation. ${ }^{25}$ The specificity of changes in functional connectivity to the feedback group (and absence of these changes in the sham subjects) indicates that it was not a result simply of repeated exposure to contamination-related images, or of practice effects, but was contingent upon the receipt of accurate, subject-specific NF.

By correlating changes in resting-state connectivity with changes in anxiety, it is possible to probe the neural basis of anxiety control. We found a negative correlation between OFC global connectivity and improved anxiety regulation, suggesting a critical role for this region in controlling contamination anxiety and confirming that we have targeted an appropriate region of the brain with our intervention (Figure 4). The correlation between increased connectivity in the right lateral parietal region and improved control over anxiety was not predicted, but it is interesting to note that this area has been implicated in OCD. ${ }^{28}$

Susceptibility artifacts make the OFC a difficult region to image. However, using an optimized pulse sequence, we were able to successfully train individuals to reorganize their brain patterns so as to better control activity in this region. This has implications beyond this study and beyond the management of anxiety, as this critical limbic area has been implicated in many different disorders involving disrupted emotional processing, including conduct disorder, bipolar disorder and addiction. ${ }^{29}$

Despite a growing literature on rt-fMRI NF, there is, to date, limited evidence that it can induce persistent changes in brain function. Most NF studies have not included a follow-up imaging session. At least two previous studies have reported persistent alterations in behavioral measures days after NF, ${ }^{30,31}$ supporting the view that rt-fMRI NF can induce a lasting reorganization of brain function. On the other hand, one study that identified changes in brain function during the NF found that those changes did not persist after the feedback. ${ }^{3}$ Two factors may have contributed to the more persistent changes in resting-state connectivity in the current study. First, by having two feedback sessions spaced several days apart, subjects in this study had a chance to consolidate their learning overnight; sleep is a critical part of some forms of learning ${ }^{32}$ and may have enhanced the persistence of plastic changes in this case. Second, anxiety-related brain regions may be more subject to lasting changes than other brain circuits. More studies are needed examining the persistence of changes induced by rt-fMRI NF in a variety of contexts to clarify these issues.

It is possible that the neural basis of contamination anxiety in patients with OCD differs from that of healthy subjects. However, the region that we targeted (the OFC) has been reported to have elevated activation during the experience of contamination anxiety in both healthy individuals and OCD patients. ${ }^{13,14}$ Thus, it is plausible that a protocol such as this one, which uses rt-fMRI NF to train subjects to control activity in their OFC, will translate into improved control over contamination anxiety in OCD patients. A study in the patient population is needed to address this issue definitively.

As our NF protocol was limited to two sessions, we are not able to identify the optimal number of sessions for this intervention. Our results suggest that two sessions are better than one: the subjects in our study did not show a significant increase in control over their OFC after a single session, but 


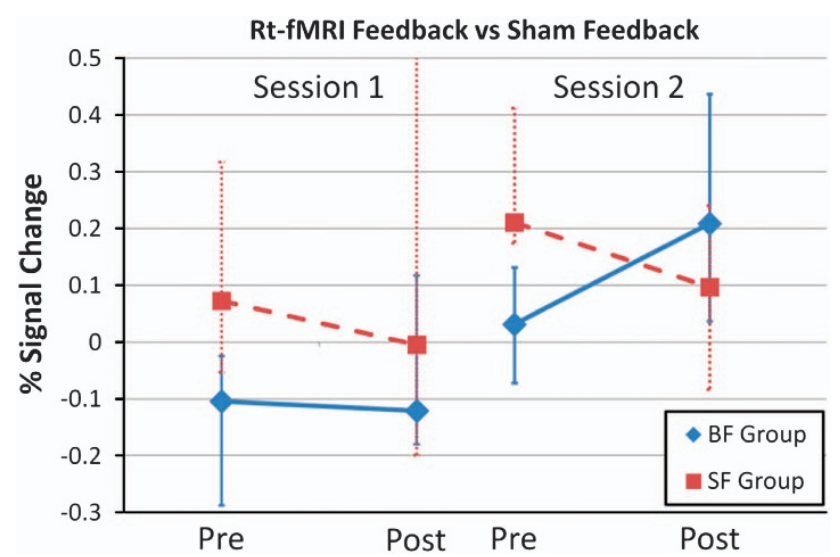

Figure 5 Control over the orbitofrontal cortex target area at the four different time points when control over the brain area was assessed. The median and quartile ranges are shown for each of the two groups at each time point. BF, biofeedback; $\mathrm{SF}$, sham feedback.

they did improve with two NF sessions (Figure 5). It is possible that a larger number of NF sessions would be able to produce still greater control of OFC activity. Future work should include studies aimed at optimizing the number of NF sessions.

Rt-fMRI NF was used to reorganize the intrinsic functional brain patterns of subjects so as to allow them to gain greater control over their contamination anxiety. The alterations lasted for several days after the completion of NF training. These results have implications for mechanism, as they imply brain plasticity rather than just a state change induced in the short term by the feedback experience. More generally, these data indicate that rt-fMRI NF can produce persistent changes in the brain circuitry underlying maladaptive behavioral states and thereby support its potential a treatment for neuropsychiatric disease.

\section{Conflict of interest}

The authors declare no conflict of interest.

Acknowledgements. This work was supported by funding from the National Institutes of Health (MH090384, EB012969, MH081190) and the Doris Duke Charitable Foundation. We thank Maolin Qiu, Jitendra Bhawnani and Hedy Sarofin for technical assistance and the State of Connecticut for its support of the Ribicoff Research Facilities at the Connecticut Mental Health Center.

1. Greenberg BD, Rauch SL, Haber SN. Invasive circuitry-based neurotherapeutics: stereotactic ablation and deep brain stimulation for OCD. Neuropsychopharmacology 2010; 35: 317-336.

2. Ruscio AM, Stein DJ, Chiu WT, Kessler RC. The epidemiology of obsessive-compulsive disorder in the National Comorbidity Survey Replication. Mol Psychiatry 2010; 15: 53-63.

3. Hamilton JP, Glover GH, Hsu JJ, Johnson RF, Gotlib IH. Modulation of subgenual anterior cinqulate cortex activity with real-time neurofeedback. Hum Brain Mapp 2011; 32: 22-31.

4. Yoo S-S, Jolesz FA. Functional MRI for neurofeedback: feasibility study on a hand motor task. NeuroReport 2002; 13: 1377-1381.

5. Zotev V, Krueger F, Phillips R, Alvarez RP, Simmons WK, Bellgowan $P$ et al. Selfregulation of amygdala activation using real-time fMRI neurofeedback. PLoS One 2011; 6 : e24522.

6. Caria A, Sitaram R, Veit R, Begliomini C, Birbaumer N. Volitional control of anterior insula activity modulates the response to aversive stimuli. A real-time functional magnetic resonance imaging study. Biol Psychiatry 2010; 68: 425-432.

7. Li X, Hartwell KJ, Borckardt J, Prisciandaro JJ, Saladin ME, Morgan PS et al. Volitional reduction of anterior cingulate cortex activity produces decreased cue craving in smoking cessation: a preliminary real-time fMRI study. Addict Biol 2012 (e-pub ahead of print).
8. Rota G, Sitaram R, Veit R, Erb M, Weiskopf N, Dogil G et al. Self-regulation of regional cortical activity using real-time fMRl: the right inferior frontal gyrus and linguistic processing. Hum Brain Mapp 2009; 30: 1605-1614.

9. Ruiz S, Lee S, Soekadar SR, Caria A, Veit R, Kircher T et al. Acquired self-control of insula cortex modulates emotion recognition and brain network connectivity in schizophrenia. Human Brain Mapp 2011; 34: 200-212.

10. deCharms RC, Maeda F, Glover GH, Ludlow D, Pauly JM, Soneji D et al. Control over brain activation and pain learned by using real-time functional MRI. Proc Natl Acad Sci 2005; 102: $18626-18631$.

11. Subramanian L, Hindle JV, Johnston S, Roberts MV, Husain M, Goebel R et al. Real-time functional magnetic resonance imaging neurofeedback for treatment of Parkinson's disease. J Neurosci 2011; 31: 16309-16317.

12. Hampson M, Scheinost D, Qiu M, Bhawnani J, Lacadie C, Leckman JF et al. Biofeedback from the supplementary motor area reduces functional connectivity to subcortical regions. Brain Connectivity 2011; 1: 91-98.

13. Mataix-Cols D, Cullen S, Lange K, Zelaya F, Andrew C, Amaro E et al. Neural correlates of anxiety associated with obsessive-compulsive symptom dimensions in normal volunteers. Biol Psychiatry 2003; 53: 482-493.

14. Mataix-Cols D, Wooderson S, Lawrence N, Brammer MJ, Speckens A, Phillips ML. Distinct neural correlates of washing, checking, and hoarding symptom dimensions in obsessivecompulsive disorder. Arch Gen Psychiatry 2004; 61: 564-576.

15. Burns GL, Keortge SG, Formea GM, Sternberger LG. Revision of the Padua Inventory of obsessive compulsive disorder symptoms: distinctions between worry, obsessions, and compulsions. Behav Res Ther 1996; 34: 163-173.

16. Hampson M, Stoica T, Saksa J, Scheinost D, Qiu M, Bhawnani $J$ et al. Real-time fMRI biofeedback targeting the orbitofrontal cortex for contamination anxiety. J Vis Exp 2012 pii 3535 .

17. Scheinost D, Hampson M, Qiu M, Bhawnani J, Constable RT, Papademetris XA. Graphics processing unit accelerated motion correction algorithm and modular system for real-time fMRI. Neuroinformatics 2013 (e-pub ahead of print).

18. Joshi A, Scheinost D, Okuda H, Belhachemi D, Murphy I, Staib LH et al. Unified framework for development, deployment and robust testing of neuroimaging algorithms. Neuroinformatics 2011; 9: 69-84.

19. Martuzzi R, Ramani R, Qiu M, Rajeevan N, Constable RT. Functional connectivity and alterations in baseline brain state in humans. Neuroimage 2010; 49: 823-834.

20. Rubinov M, Sporns $\mathrm{O}$. Complex network measures of brain connectivity: uses and interpretations. Neuroimage 2010; 52: 1059-1069.

21. Buckner RL, Vincent JL. Unrest at rest: default activity and spontaneous network correlations. Neurolmage 2007; 37: 1091-1096.

22. Hampson M, Tokoglu F, Shen X, Scheinost D, Papademetris X, Constable RT. Intrinsic brain connectivity related to age in young and middle aged adults. PloS One 2012; 7: e44067.

23. Cole MW, Yarkoni T, Repovs G, Anticevic A, Braver TS. Global connectivity of prefrontal cortex predicts cognitive control and intelligence. J Neurosci 2012; 32: 8988-8999.

24. Van Dijk KR, Sabuncu MR, Buckner RL. The influence of head motion on intrinsic functional connectivity MRI. Neuroimage 2012; 59: 431-438.

25. Ochsner KN, Gross JJ. Cognitive emotion regulation: Insights from social cognitive and affective neuroscience. Curr Dir Psychol Sci 2008; 17: 153-158.

26. deCharms RC, Christoff K, Glover GH, Pauly JM, Whittield S, Gabrieli JDE. Learned regulation of spatially localized brain activation using real-time fMRI. Neurolmage 2004; 21: 436-443.

27. Rota G, Handjaras G, Sitaram R, Birbaumer N, Dogil G. Reorganization of functional and effective connectivity during real-time fMRI-BCl modulation of prosody processing. Brain Lang 2011; 117: 123-132.

28. Menzies L, Chamberlain SR, Laird AR, Thelan SM, Sahakian BJ, Bullmore ET. Integrating evidence from neuroimaging and neuropsychological studies of obsessive-compulsive disorder: the orbitofrontal-striatl model revisited. Neurosci Biobehav Rev 2008; 32: 525-549.

29. Jackowski AP, Filho GM, Almeida AG, Araujo CM, Reis M, Nery F et al. The involvement of the orbitofrontal cortex in psychiatric disorders: an update of neuroimaging findings. Rev Bras Psiquiatr 2012; 34: 207-212.

30. Shibata K, Watanabe T, Sasaki Y, Kawato M. Perceptual learning incepted by decoded fMRI neurofeedback without stimulus presentation. Science 2011; 334: 1413-1415.

31. Yoo SS, O'Leary HM, Fairneny T, Chen NK, Panych LP, Park H et al. Increasing cortical activity in auditory areas through neurofeedback functional magnetic resonance imaging. Neuroreport 2006; 17: 1273-1278.

32. Gais S, Born J. Low acetylcholine during slow-wave sleep is critical for declarative memory consolidation. Proc Natl Acad Sci USA 2004; 101: 2140-2144.

Translational Psychiatry is an open-access journal (c) ${ }_{\mathrm{By}} \mathrm{Na}$
licensed under a Creative Commons Attribution-NonCommercialShareAlike 3.0 Unported License. To view a copy of this license, visit http://creativecommons.org/licenses/by-nc-sa/3.0/ 